\title{
Welche Vorteile bietet das winkelsteife Implantat am proximalen Humerus?
}

\author{
Wolfgang Köstler, Peter C. Strohm, Norbert P. Südkamp
}

\section{Zusammenfassung}

Die proximale Humerusfraktur und die Humeruskopffraktur sind häufige Frakturen gerade des älteren Menschen. Für die Diagnose sind Röntgenbilder in 2 Ebenen notwendig, ggf. ist auch eine CT-Untersuchung zur Therapieentscheidung erforderlich. Die Indikation zur konservativen oder operativen Behandlung, Kopferhaltung oder prothetischem Ersatz hängt u.a. von der Frakturform, der Knochenqualität und den Präferenzen des Chirur- gen ab. Gering dislozierte Frakturen können konservativ behandelt werden, dislozierte Mehrfragmentfrakturen des Humeruskopfes können meist kopferhaltend operiert werden. Eine primäre endoprothetische Versorgung bleibt Ausnahmefällen vorbehalten. Über das ideale Implantat gerade beim osteoporotischen Knochen herrscht noch keine einheitliche Meinung. Die neuen winkelstabilen Platten bieten gerade beim osteoporotischen Knochen theoretische Vorteile.

\section{Einleitung}

Frakturen am proximalen Humerus sind gemäß der AO-Einteilung extraartikuläre Frakturen. Viel häufiger und gelegentlich in Kombination treten jedoch Humeruskopffrakturen auf. Gerade beim alten Menschen stellt diese Fraktur eine der häufigsten Verletzungen dar. Die Ergebnisse der operativen Versorgung sind häufig nicht zufriedenstellend. Die bekannten Probleme der mangelnden Implantatverankerung mit Notwendigkeit der zusätzlichen Ruhigstellung, der sekundären Implantatlockerung, das Auftreten von avaskulären Nekrosen v.a. der Kopfkalotte konnten bisher nicht ausreichend gelöst werden. Es existieren keine einheitlichen Therapiekonzepte und in letzter Zeit wurden einige neue Implantate vorgestellt, um die oben genannten Problemsituationen zu verhindern. Das Prinzip der Winkelstabilität und die verschiedenen Implantate wurden in den vorangegangenen Artikeln dieses Heftes erläutert.

OP-JOURNAL 2004; 20: 14-17

(c) Georg Thieme Verlag KG Stuttgart · New York

\section{Proximale Humerusfraktur}

Die Frakturen am proximalen Humerus ohne Beteiligung des Kopfes können bei geringer Dislokation konservativ behandelt werden. Ein Röntgenbild in 2 Ebenen ist zur Diagnosestellung ausreichend, wobei eine exakte zweite Ebene aus Schmerzgründen meist nur mithilfe abzubilden sein wird. Eine gründliche Untersuchung von Durchblutung, Motorik und Sensibilität ist selbstverständlich, auf das Vorgehen bei offenen Frakturen und Frakturen mit Zusatzverletzungen soll in diesem Rahmen nicht eingegeangen werden.

Bei größerer Dislokation kann ggf. reponiert werden, es ist unter Bildverstärker zu prüfen, ob eine ausreichende Retention der Fraktur zu erreichen ist. Wir verwenden bei konservativer Behandlung einen gipsverstärkten Gilchristverband, aus dem nach etwa 10 Tagen mit krankengymnastischen Übungen begonnen wird.

\section{Therapie}

Bei operativer Versorgung bevorzugen wir mit der Marknagelung ein gedecktes Verfahren, aus finanziellen Erwägungen bleibt die Versorgung mit dem Flexnagel speziellen Indikationen vorbehalten. Die Nagelung wird in der Regel retrograd in Bauchlage durchgeführt, nachteilig bei sehr weit proximal liegenden Brüchen ist ein erhöhtes Risiko von Nervenverletzungen bei der proximalen Verriegelung. Über Erfahrungen mit einem speziellen Marknagel für die Versorgung von proximalen Humerusfrakturen verfügen wir noch nicht.

Für die winkelstabile Versorgung bei proximalen Frakturen wurden anfangs herkömmliche Winkelplatten verwendet [4], später auch neue Plattendesigns entwickelt [6]. In unserer Klinik wird die proximale Humerusplatte (Fa. Synthes) verwendet, in leicht abgewandelter Form ist diese Platte auch als Philosplatte im Handel erhältlich. Da die Platte bisher nur relativ kurz angeboten wurde, konnten längerstreckige Frakturen damit nicht versorgt werden. In Kürze werden jedoch längere Versionen lieferbar sein (Abb.1).

Aus Gründen der Weichteilschonung sehen wir für die Plattenosteosynthese bei dieser Frakturlokalisation nur eine

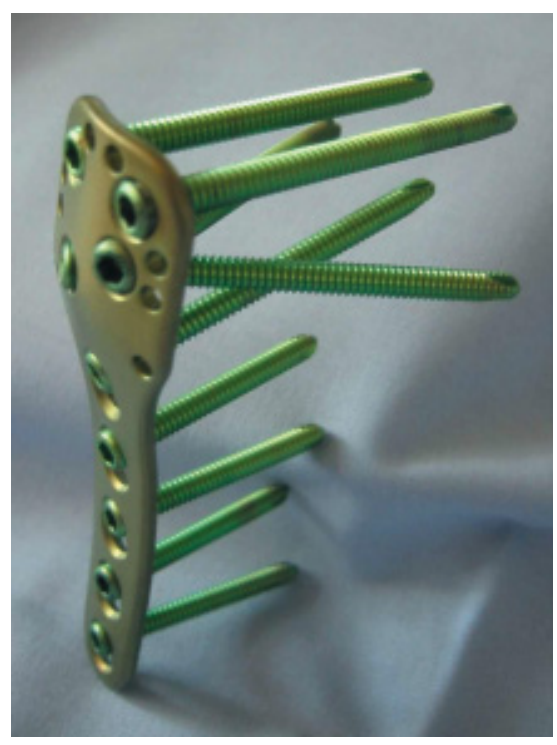

Abb.1 Proximale Humerusplatte. 
Ausnahmeindikation z.B. bei offener Fraktur. Die primäre Stabilität der herkömmlichen Marknägel ist beim jungen Menschen aus unserer Sicht ausreichend. Beim alten Menschen ist die proximale Verriegelung im Bereich des Schaftes mit bikortikaler Verankerung meist stabil. Falls die Verriegelungsbolzen bei sehr weit proximaler Fraktur jedoch im Kopfbereich zu liegen kommen, ist eine bikortikale Verankerung im Gelenkbereich nicht möglich und der Halt der Bolzen unzureichend. Eine Lockerung mit Dislokation der Bolzen ist bei diesen Patienten immer wieder festzustellen. Hier scheint eine winkelstabile Verankerung der Bolzen theoretisch von Vorteil, um das Risiko von sekundären Instabilitäten zu vermindern. Mit winkelstabilen Marknägeln haben wir bisher keine Erfahrungen, sie befinden sich aber bereits in klinischer Erprobung. Auf diese Entwicklungen wird im Artikel von Prof. Rommens noch eingegangen.

Bei Kombinationsverletzungen mit zusätzlicher Frakturkomponente im Bereich des Humeruskopfes ist bei geschlossener Reposition eine Versorgung mit Marknagel gerade bei subkapitaler Fraktur gut möglich. Zusätzliche dislozierte Frakturen des Tuberkulum majus können ggf. perkutan verschraubt werden. Bei Kalottenfrakturen und bei Notwendigkeit einer offenen Reposition kommen die Grundsätze der Versorgung von Humeruskopffrakturen zum Tragen. In der Literatur wird über gute Ergebnisse mit verschiedenen proximalen Nagelsystemen berichtet, die winkelstabile Versorgung derartiger Kombinationsverletzungen scheint zumindest theoretisch von Vorteil. Die winkelstabile Versorgung mit Platten war aus oben genannten Gründen bisher nur bei sehr weit proximal liegenden Frakturkombinationen möglich.

Studienergebnisse über die Versorgung mit winkelstabilen Systemen bei proximalen Humerusfrakturen gibt es bisher nur vereinzelt, prospektiv randomisierte Vergleichsstudien existieren nicht.

\section{Proximale Humerusfraktur}

Für die Diagnosestellung ist ein Röntgenbild in 2 Ebenen ausreichend. Gering dislozierte Frakturen können konservativ behandelt werden. An operativen Verfahren stehen als gedeckte Osteosynthese die Marknagelung und als offenes Verfahren die Plattenosteosynthese zur Verfügung. V.a. beim osteoporotischen Knochen versprechen die winkelstabilen Osteosyntheseverfahren eine geringere Komplikationsrate.

\section{Humeruskopffrakturen}

Gerade beim alten Menschen stellen diese Frakturen oft eine Herausforderung dar. Die Altersgruppe der über 60-Jährigen stellt über $70 \%$ der betroffenen $\mathrm{Pa}$ tienten [1]. Stabile (unter Bildverstärker zu prüfen!) gering dislozierte Frakturen können konservativ behandelt werden. Im Subakromialbereich können jedoch auch geringe Stufenbildungen zu funktionellen Einbußen oder Schmerzen führen. Eine differenzierte Abhandlung über die verschiedenen Aspekte bei Humeruskopffrakturen sprengt den Rahmen dieses Beitrages, es wird dazu auf verschiedene in jüngster Zeit publizierte Arbeiten verwiesen $[3,5,7,9]$.

Die Diagnosestellung erfordert eine axiale Aufnahme, die dazu notwendige Abduktion von etwa 70 Grad ist bei gehaltenem Arm fast immer möglich. Sowohl die Beteiligung des Tuberkulum minus, als auch die Beurteilung der Kopf-SchaftAchse in der Sagittalebene ist mit dieser Aufnahme möglich. Falls z. B. eine Fraktur der Kopfkalotte („head split“) nicht ausgeschlossen werden kann, ist eine CT-Untersuchung anzuraten.

\section{Therapie}

Die Vielzahl der Therapieempfehlungen in der Literatur [9] von konservativer Behandlung bis zur primären endoprothetischen Versorgung verdeutlicht den Mangel an sicheren Daten. Die Entwicklung der winkelstabilen Implantate verändert selbstverständlich nichts an den biologischen Gegebenheiten in der Fraktursituation. Die primäre Durchblutungssituation der Frakturfragmente kann nicht beeinflusst, ja nicht einmal mit diagnostischen Mitteln sicher abgeschätzt werden. Informationen wie der Abstand des Schaftes von der Kalotte v.a. medial können nur Anhaltspunkte bieten (Abb.2).

Im biomechanischen Bereich mit den bekannten Problemen der schwierigen Implantatverankerung, gerade beim osteoporotischen Knochen, und des sekundären Repositionsverlustes mit Implantatversagen, sind von den neuen Entwicklungen verschiedener winkelstabiler Osteosyntheseverfahren Verbesserungen zu erwarten.

\section{Winkelstabile Plattenosteosynthese}

Die winkelstabilen Plattensysteme wurden bereits genannt, die von Hessmann [2] beschriebene schonende Repositions-

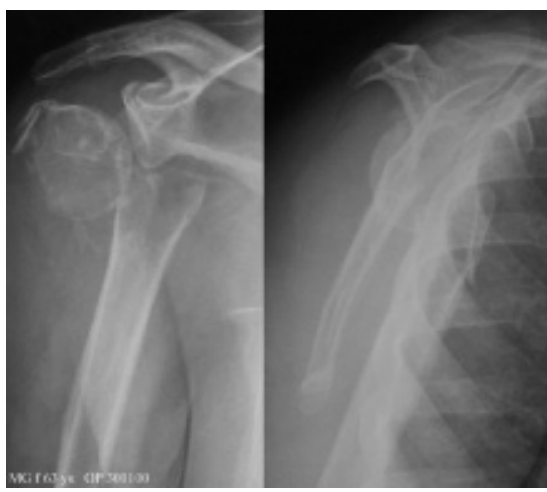

Abb. 2 Dislozierte subkapitale Humerusfraktur.

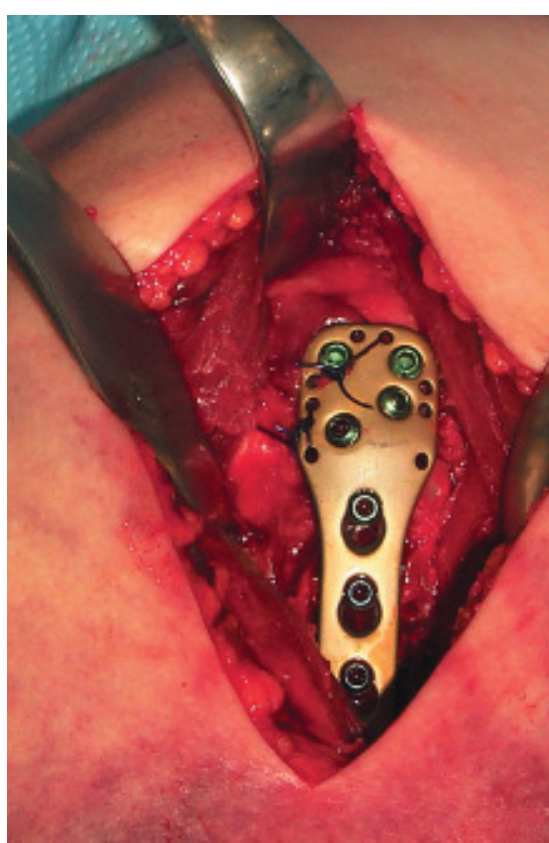

Abb.3 Intraoperativer Situs mit PH-Platte.

technik ist zur Vermeidung von zusätzlichen iatrogenen Schäden an der Durchblutung dringend zu empfehlen. Nach Darstellung über einen deltoideopectoralen Zugang mit Lateralisierung der V. cephalica, erfolgt in Abduktionsstellung des Armes die indirekte Reposition mit Raspatorium und die Fixation mit K-Drähten. Nach Kontrolle mit Bildverstärker in beiden Ebenen wird ggf. erneut reponiert. Die PHP wird angelegt und im Schaftbereich mit einer Schraube fixiert.

Die Platte muss als interner Fixateur nicht unbedingt am Knochen anliegen, ggf. kann es dadurch sogar zu einer Medialisierung des Kopfes kommen. Nach Kontrolle im Bildverstärker, ein kraniales Überstehen über dem Tuberkulum majus ist wie bei allen Plattenosteosynthesen zu vermeiden, erfolgt die Fixation im Kopfbereich mit winkelstabilen Schrauben. 


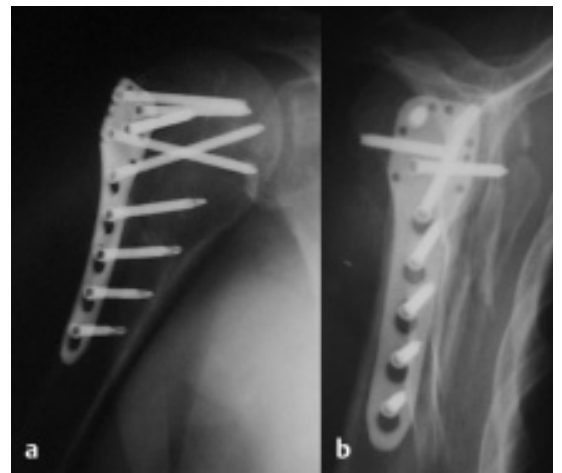

Abb. 4a u.b Postoperatives Röntgenbild.

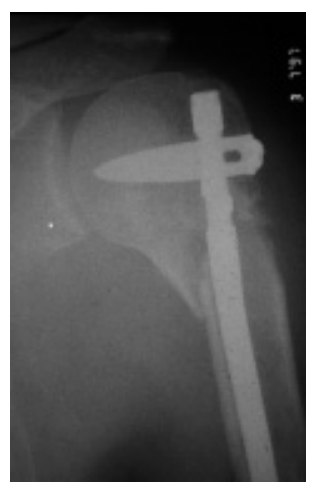

Abb. 5 Proximaler Humerusnagel.

Die Bohrung erfolgt im Unterschied zum Kleinfragmentinstrumentarium hier mit einem 2,8-mm-Bohrer (Abb.3).

Die divergente Anordnung im Kopfbereich dient der Fixation der Tuberkula und erhöht die Ausreißkraft im Kopf. Bezüglich der Schraubenlänge ist eine genaue Kontrolle mit Bildverstärker unter Drehen des Humeruskopfes erforderlich. Um ein Durchtreten der Schrauben durch sekundäre Sinterung der Kalotte zu vermeiden, sollte diese nicht durchbohrt werden. Andererseits finden zu kurz gewählte Schrauben keinen ausreichenden Halt im subchondralen Knochen. Die Schrauben werden mit einem Drehmomentschlüssel und $1,5 \mathrm{Nm}$ fixiert. Im Schaftbereich sind 3 Schrauben ausreichend, eine Fixation der Tuberkula mit zusätzlichen Nähten ist an separaten Plattenlöchern möglich.

Im Unterschied zu konventionellen Plattenosteosynthesen ist eine taktile Kontrolle des Schraubensitzes beim Eindrehen nicht möglich. In den Röntgenbildern zeigen sich die Schrauben nicht immer im idealen Winkel eingebracht, ein Abweichen von mehr als 5-10 Grad vom idealen Winkel führt jedoch zu einer deutlich reduzierten Haltekraft der Schrauben.

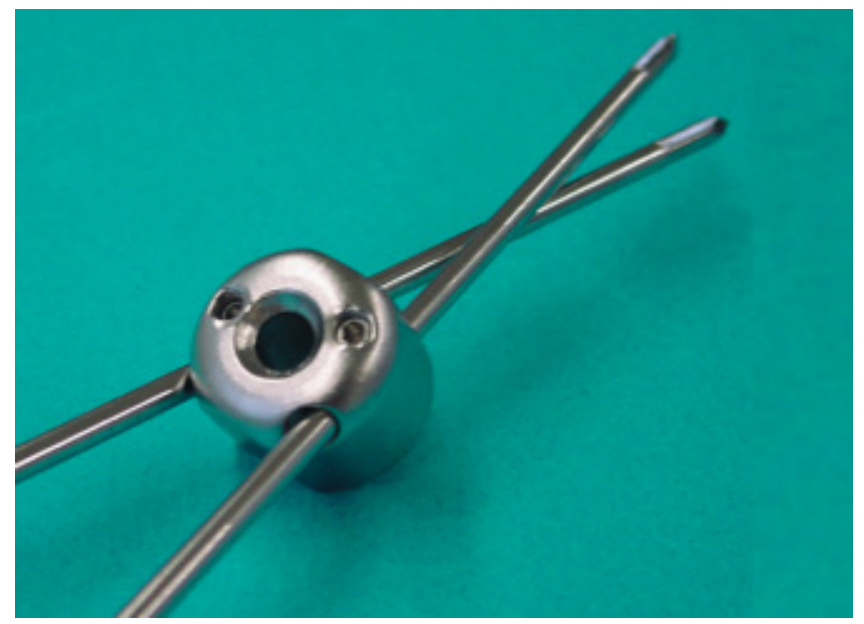

Abb. 6 Humerusblock.

Der Drehmomentschlüssel kann in diesen Fällen einen festen Sitz des Schraubenkopfes in der Platte suggerieren, der jedoch durch ein Verklemmen des Schraubenkopfes im Gewinde des Plattenloches bedingt ist. Es sollte auch erwähnt werden, dass sich die Metallentfernung gerade bei nicht sorgfältig behandeltem Inbus manchmal schwierig gestaltet (Abb.4a,b).

In der klinischen Anwendung hat sich die Platte bei uns bewährt, die Nachuntersuchung der ersten 100 operierten Patienten wird gerade durchgeführt, außerdem wird eine prospektive Untersuchung im Rahmen einer AO-Studie durchgeführt.

In der Nachbehandlung sehen wir aber auch bereits eine Reihe von Komplikationen. Implantatlockerungen können trotz winkelstabiler Verankerung auftreten, sowohl im Kopf- als auch im Schaftbereich. Vereinzelt sahen wir auch Plattenbrüche am Übergang zum Schaft, außerdem sind bei einzelnen Patienten Schrauben durch Sinterung der Kalotte in das Gelenk ausgetreten und zwangen zum Wechsel oder zur vorzeitigen Metallentfernung.

Ob sich die theoretischen Vorteile der winkelstabilen Platten in besseren klinischen Ergebnissen widerspiegeln, bleibt im Moment noch offen.

\section{Humeruskopffrakturen}

Zur exakten Einschätzung der Fraktur ist eine CT Untersuchung hilfreich. Die Therapieempfehlungen in der Literatur sind uneinheitlich. Falls ein operatives Vorgehen gewählt wird, sollte bei offenem Vorgehen eine übungsstabile Osteosynthese erreicht werden. Gedeckte Verfahren zeigen auch nach 2-3 wöchiger Ruhigstellung vergleichbare Ergebnisse.

\section{Verriegelungsnagel}

Eigene Erfahrungen mit winkelstabilen Marknägeln haben wir nicht. Auf die theoretischen Vorteile gerade bei Kombinationsverletzungen von Schaft- und proximalen Frakturen, bzw. proximalen Frakturen und Humeruskopffrakturen wurde bereits hingewiesen. Hier werden sicher in Kürze neue Implantate vorgestellt und der klinischen Prüfung unterzogen (Abb.5).

\section{Humerusblock}

Eine weitere zumindest z.T. winkelstabile Osteosynthesetechnik stellt die von Resch [8] vorgestellte Versorgung mit dem Humerusblock dar. Ein Vorteil dieser Technik liegt in der minimal-invasiven Anwendungstechnik (Abb.6).

Zwei gekreuzt eingebrachte $2,5 \mathrm{~mm}$ starke Kirschner-Drähte werden nach Reposition über ein Führungsinstrumentarium eingebracht und mit Klemmschrauben im Humerusblock fixiert.

Dieser ist mit einer Kleinfragmentschraube am Schaft fixiert. Die Drähte können somit nicht wandern und sind auch für die Rotation stabil. Durch den Valguswinkel, in dem die Drähte eingebracht sind, ist eine relativ axiale Belastung der Drähte vorgegeben, was das Risiko der Valgusdislokation der Kalotte mindert. Allenfalls ein Heraustreten der Drähte aus der Kalotte kann auftreten, diese sollten dann vor der Mobilisierung entfernt werden (Abb. 7).

Durch das geschlossene Vorgehen werden die Gleitschichten nicht eröffnet, eine Ruhigstellung von etwa 3 Wochen führt deshalb nicht zwangsweise zu einem Einsteifen der Schulter. Bei Beteili- 


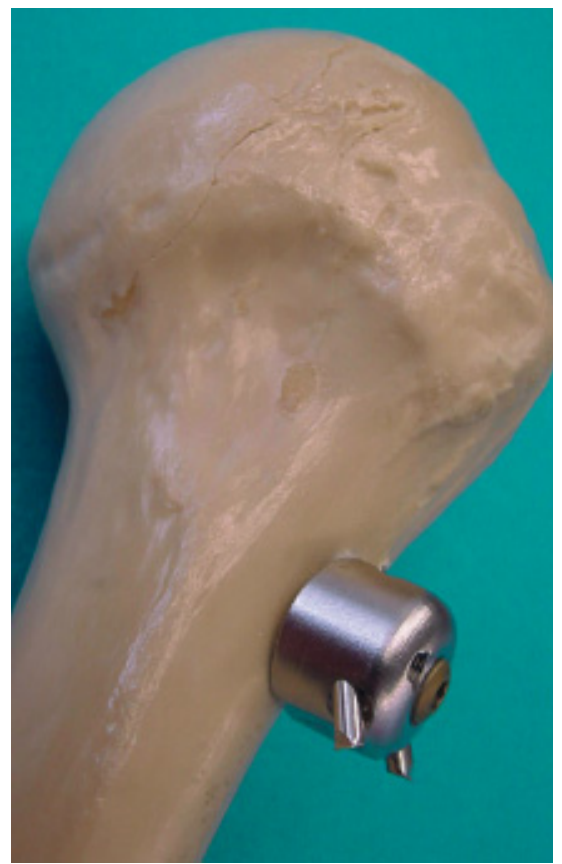

Abb.7 Humerusblock am Knochenmodell.

gung des Tuberkulum majus mit Dislokation ist eine zusätzliche perkutane Verschraubung z.B. mit kanülierten Kleinfragmentschrauben $(4,0)$ erforderlich. Wir haben diese Technik bisher nur in Einzelfällen angewandt, Resch berichtet über gute Ergebnisse. Erfahrungen anderer Anwender liegen bisher nicht vor (Abb. 8).

\section{Schlussfolgerung}

Die neuen winkelstabilen Osteosyntheseverfahren zeigen theoretische Vorteile. Ob die höheren Kosten durch bessere klinische Ergebnisse gerechtfertigt sind, muss noch bewiesen werden.

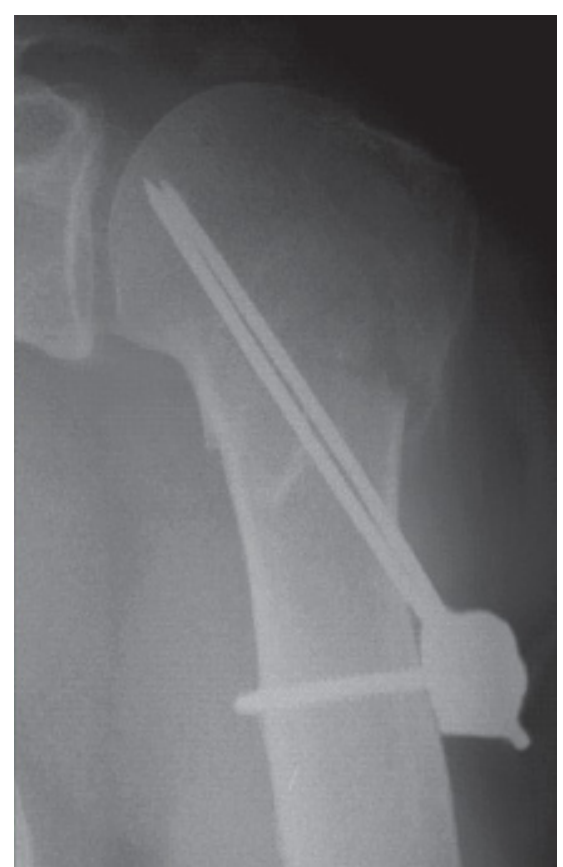

Abb. 8 Postoperatives Röntgenbild mit Humerusblock.

\section{Schlussfolgerung}

Die Vielzahl der Therapieempfehlungen gerade bei Humeruskopffrakturen zeigt, dass eine Standardbehandlung für die verschiedenen Frakturformen noch nicht existiert. Insbesondere beim alten Menschen mit osteoporotischer Knochenstruktur traten bei osteosynthetischer Versorgung häufig Komplikationen wie sekundärer Repositionsverlust und Implantatversagen auf. Auch Kombinationsverletzungen oder langstreckige Frakturen sind mit den konventionellen Osteosyntheseverfahren gelegentlich nur schwer übungsstabil zu versorgen. Die winkelstabile Plattenosteosynthese einem internen Fixateur entsprechend zeigt theoretische Vorteile gerade beim osteoporotischen Knochen. Ob diese sich in besseren klinischen Ergebnissen widerspiegeln, muss sich erst erweisen.

\section{Literatur}

1 Court-Brown CM, Garg A, Mc Queen MM. The epidemiology of proximal humeral fractures. Acta Orthop 2001; 72: 365-371

2 Hessmann MH, Rommens PM. Osteosynthesetechniken bei proximalen Humerusfrakturen. Chirurg 2001; 72: 1235-1245

3 Köstler W, Strohm PC, Südkamp NP. Die proximale Humerusfraktur im hohen Lebensalter. Chirurg 2003; 74: 985-989

4 Kohler A, Simmen HP, Duff C, Kossmann T, Trentz O. Osteosynthese der subkapitalen Humerusfraktur mit unkonventionellen Implantaten. Helv Chir Acta 1992; 59: 679680

5 Lill H, Josten C. Konservative oder operative Versorgung der Humeruskopffraktur beim alten Menschen. Chirurg 2001; 72: $1224-$ 1234

${ }^{6}$ Mückter H, Herzog I, Becker M, Vogel W, Meeder PJ, Buchholz J. Die winkel- und rotationsstabile Osteosynthese proximaler $\mathrm{Hu}-$ merusfrakturen mit der Humerus Fixateurplatte. Chirurg 2001; 72: 1327-1335

7 Resch H. Die Humeruskopffraktur. Unfallchirurg 2003; 106: $602-617$

8 Resch H, Povacz P, Fröhlich H, Wambacher M. Percutaneous fixation of three- and four-fractures of the proximal humerus J Bone Joint Surg Br 1997; 79: 295-300

9 Tingart M, Bäthis H, Bouillon B, Tilling TH. Die dislozierte proximale Humerusfraktur: Gibt es gesicherte Therapiekonzepte? Chirurg 2001; 72: 1284-1291

\section{Dr. med. Wolfgang Köstler Oberarzt}

\section{Dr. med. Peter C. Strohm}

Oberarzt

Prof. Dr. med. Norbert P. Südkamp

Ärztlicher Direktor

Department für Orthopädie und

Traumatologie

Albert-Ludwigs-Universität

Hugstetter Str. 55

D-79106 Freiburg 